\title{
MicroRNA-1290 promotes asiatic acid-induced apoptosis by decreasing BCL2 protein level in A549 non-small cell lung carcinoma cells
}

\author{
KI BBEUM KIM $^{1 *}$, KARAM KIM ${ }^{1 *}$, SEUNGHEE BAE ${ }^{1}$, YEONGHMIN CHOI $^{1}$, HWA JUN CHA ${ }^{1}$, \\ SOO YEON KIM ${ }^{1}$, JAE HO LEE ${ }^{2}$, SO HYEON JEON ${ }^{3}$, HO JUNG JUNG ${ }^{4}$, \\ KYU JOONG AHN ${ }^{4}$, IN-SOOK AN ${ }^{1}$ and SUNGKWAN AN ${ }^{1}$
}

\footnotetext{
${ }^{1}$ Molecular-Targeted Drug Research Center and Korea Institute for Skin and Clinical Sciences, Konkuk University, Seoul 143-701; ${ }^{2}$ Laboratory of Molecular Oncology, Cheil General Hospital and Women's Healthcare Center,

Kwandong University, College of Medicine, Seoul 100-380; ${ }^{3}$ Department of Beauty Design, Dongwon University, Gwangju, Gyeonggi-do, 464-711; ${ }^{4}$ Department of Dermatology, Konkuk University School of Medicine, Seoul 143-701, Republic of Korea
}

Received April 17, 2014; Accepted June 11, 2014

DOI: $10.3892 /$ or.2014.3319

\begin{abstract}
Asiatic acid, a triterpenoid derived from Centella asiatica, is a putative anticancer agent in several types of cancer cells. Investigations of its biological role in negative regulation of cell growth have focused on the extent of induction of apoptosis in a cell-type-specific manner. In this study, we identified an important regulator of asiatic acid-induced cell death, microRNA (miR)-1290, which sensitizes cells to asiatic acid-induced cytotoxicity and negatively regulates BCL2 expression. Asiatic acid significantly upregulated miR-1290, and asiatic acid-induced cell death was shown to be dependent on miR-1290 activity. Molecular assays demonstrated that BCL2 mRNA is a direct target of miR-1290-mediated RNA interference. The results of functional studies suggest that miR-1290 suppresses cell viability and cell cycle progression. These data provide insight into miR-1290-mediated cellular mechanisms in asiatic acid-treated A549 non-small cell lung carcinoma cells.
\end{abstract}

\section{Introduction}

Centella asiatica (C. asiatica) is used as a traditional medicine in Asia (1). Four classes of triterpenoids are present in C. asiatica extract: asiatic acid, madecassic acid, asiaticoside,

Correspondence to: Professor Sungkwan An, Molecular-Targeted Drug Research Center and Korea Institute for Skin and Clinical Sciences, Konkuk University, 120 Neungdong-ro, Gwangjin-gu, Seoul 143-701, Republic of Korea

E-mail: ansfgrc@konkuk.ac.kr

${ }^{*}$ Contributed equally

Key words: asiatic acid, A549 cells, microRNA, BCL2, apoptosis and madecassoside (2). Asiaticoside, the most abundant triterpene glycoside in the extract, is converted to asiatic acid by hydrolysis in vivo (3). Asiatic acid functions as an antioxidant and antitoxic agent in normal cells, and as an anticancer agent in cancer cells (4-8). Several studies have shown that pretreatment with asiatic acid has a protective effect against $\mathrm{H}_{2} \mathrm{O}_{2}$-induced cytotoxic damage in neuronal cells and against D-galactosamine/lipopolysaccharide-induced toxicity in hepatocytes $(4,5)$. However, various reports have also shown that asiatic acid mediates a variety of anticancer effects as a result of growth inhibition and apoptosis in several types of cancer cells, such as HepG2 human hepatoma, HT-29 human colon adenocarcinoma, and MCF7 human breast cancer cells (6-8). Although the role of asiatic acid in cancer cell death has been widely studied, asiatic-mediated cellular mechanisms are less well known.

Apoptosis, or programmed cell death, is characterized by several morphological and biochemical changes, including reduced cell volume, condensation of nuclear chromatin, DNA fragmentation, and an increased proportion of sub-G1 phase cells (9). Initiation of apoptotic processes in cells induces activation of proapoptotic proteins, as well as inactivation of antiapoptotic proteins (10). B cell CLL/lymphoma 2 (BCL2) family proteins are involved in the regulation of apoptosis either as death antagonists or death agonists (11). Of these, BCL2 is a critical regulator of cell growth and antiapoptotic processes. Under normal physiological conditions, BCL2 binds to the proapoptotic proteins BAX and BAK to prevent mitochondrial outer membrane permeabilization and cytochrome $c$ release into the cytosol. Many tumor cells overexpress antiapoptotic BCL2 and become resistant to chemotherapy and radiotherapy (11). Recently, asiatic acid was reported to induce apoptosis in several types of cancer cell, and to affect the level of expression of BCL2 and BCL-XL $(7,8)$. However, the mechanisms underlying asiatic acid-induced downregulation of BCL2 are largely unknown. 
MicroRNAs (miRNAs) are small RNAs, 19-25 nucleotides in length, that play important roles in growth, differentiation and cell death (12). Various oncogenic miRNAs (oncomiRs) and tumor suppressor miRNAs have been identified. For example, miR-34a inhibits oncogenic cellular transformation by suppressing oncogenes, such as proto-oncogene c-Met, cyclin-dependent kinase 4 (CDK4), and BCL2 (13-15). miR-21, which is a well-studied oncomiR, also targets many key tumor suppressor genes, such as phosphatase and tensin homolog (PTEN), programmed cell death 4 (PDCD4), and TP63 (16-19). Recently, miR-21 was implicated in the phenomenon of cancer cell addiction to key oncogenes (20). This finding strongly indicates that an examination of miRNA-based cellular mechanisms is critical to an understanding of tumorigenesis and anticancer processes, and certain miRNAs may be critical targets for anticancer therapy. We previously reported that treatment of human cells with C.asiatica extract altered miRNA expression profiles $(21,22)$, indicating that the effects of the extract on cells might involve miRNA-specific mechanisms.

In the present study, using miRNA microarray, we showed that asiatic acid alters expression profiles of specific miRNAs in A549 non-small cell lung carcinoma (NSCLC) cells. We also found that miR-1290 is the most extensively upregulated miRNA, and that asiatic acid-induced anticancer activity is dependent on the expression of this miRNA. We further demonstrated that miR-1290 directly targets $B C L 2$ mRNA in the cell.

\section{Materials and methods}

Cell lines, RNA oligonucleotide and chemicals. A549 NSCLC cells (American Type Culture Collection, Manassas, VA, USA) were grown in RPMI-1640 (Gibco; Life Technologies, Grand Island, NY, USA) supplemented with $10 \%$ fetal bovine serum (Sigma-Aldrich, St. Louis, MO, USA), $100 \mathrm{U} / \mathrm{ml}$ penicillin, and $100 \mu \mathrm{g} / \mathrm{ml}$ streptomycin. Asiatic acid (Sigma-Aldrich) was dissolved in DMSO and stock in 25, 50, 75, and $100 \mathrm{mM}$. To evaluate the cytotoxicity of asiatic acid, A549 cells were seeded in 96-well plates at a density of $4 \times 10^{4}$ cells/well. miR-1290 mimic, antagomiR-1290, and negative control miRNA were purchased from Qiagen (Hilden, Germany). All miRNAs were resuspended in nuclease-free water (USB; Affymetrix, Santa Clara, CA, USA) to a final stock concentration of $100 \mu \mathrm{M}$. miRNA transfection was performed in serum-free medium with Lipofectamine RNAiMAX (Invitrogen; Life Technologies) for $6 \mathrm{~h}$. Medium was replaced with fresh medium, and the cells were incubated for $24 \mathrm{~h}$.

Cell viability assay. The cytotoxicity of asiatic acid in A549 cells was evaluated using a water-soluble tetrazolium salt (WST-1) assay (EZ-Cytox Cell Viability Assay Kit; Itsbio, Seoul, Korea). WST-1 solution was added to cultures at a volume $10 \%$ that of the culture medium, and cells were incubated at $37^{\circ} \mathrm{C}$ for $0.5 \mathrm{~h}$. Cell viability was evaluated by measuring the absorbance at $450 \mathrm{nM}$ using an iMark microplate reader (Bio-Rad, Hercules, CA, USA). All results are presented as the mean percentage \pm standard deviation (SD) of three independent experiments. A P-value of $<0.005$ (or $<0.01$ ), as determined by the Student's t-test, was considered to indicate a statistically significant difference.
Analysis of cell cycle by flow cytometry. Cells were collected and fixed in cold $70 \%$ ethanol at $4^{\circ} \mathrm{C}$ for $1 \mathrm{~h}$. Fixed cells were then stained by incubation with propidium iodide (PI) staining solution [50 $\mu \mathrm{g} / \mathrm{ml}$ PI, $0.5 \%$ Triton X-100 (both from SigmaAldrich), and $100 \mu \mathrm{g} / \mathrm{ml} \mathrm{RNase]} \mathrm{at} 37^{\circ} \mathrm{C}$ for $1 \mathrm{~h}$. Changes in cell cycle were determined based on evaluation of the intensity of fluorescent PI staining of 10,000 cells using the FL2-H channel of a FACSCalibur (BD Biosciences, San Jose, CA, USA).

Construction of 3'-UTR reporter plasmid and luciferase assay. To construct luciferase reporter plasmids, target fragments were ligated into the $X b a \mathrm{I}$ site downstream of the luciferase gene in the pGL3-promoter vector (Promega, Madison, WI, USA). The region (+2776 to +3286) in the human BCL2 gene containing miR-1290 recognition site was amplified by PCR using the following primers adapted to the XbaI site: 5'-CTAGTCTAGACTAGGTGACAGTTATATCTGTTG TCC-3' and 5'-CTAGTCTAGACTAGCCACGTGGAGCATA CTGC-3'. DNA sequence analyses confirmed the nucleotides sequence of the constructed plasmid (pGL3-BCL2-3'-UTR). For the luciferase assays, pGL3 and pGL3-BCL2-3'-UTR plasmids were transiently transfected with negative control miRNA, miR-1290 or antagomiR-1290 mimics and the pSV- $\beta$-galactosidase (pSV- $\beta$-gal) plasmid into the cells. Then, luciferase activity and $\beta$-galactosidase activity were assayed as described previously (36). Briefly, after transfection, the cells were re-suspended in Passive Lysis Buffer (Promega Corp., Madison, WI, USA), and the luciferase activity was measured with a Veritas Luminometer (Turnur Designs, Sunnyvale, CA, USA). $\beta$-galactosidase activity was measured using Luminescent $\beta$-galactosidase Detection Kit II (Clontech Laboratories, Inc., Mountain View, CA, USA) according to the manufacturer's protocol. The relative luciferase activity was normalized to $\beta$-galactosidase activity. The results are the averages of three independent experiments.

Isolation of total RNA and quantitative real-time PCR analysis. Total RNA was isolated using TRIzol reagent (Life Technologies) according to the manufacturer's protocol. The purity and concentration of the RNA was evaluated based on the OD at $260 \mathrm{~nm}$ and the OD 260/230 and 260/280 ratios using MaestroNano ${ }^{\circledR}$, a micro-volume spectrophotometer (Maestrogen, Las Vegas, NV, USA). The recommended parameters of RNA quality for cDNA synthesis are OD 260/230 >1.8 and OD 260/280 ratio in the range of 1.8-2.0. cDNAs for sensitive and specific miRNA detection were synthesized using the miScript II RT Kit (Qiagen) according to the manufacturer's protocol. Quantitative real-time PCR was performed using the miR-1290-specific primer, Hs_miR-1290_1 miScript Primer Assay (Qiagen), and U6 snRNA-specific primer, Hs_RNU6-2_11 miScript Primer Assay (Qiagen). PCR was performed using the miScript SYBR-Green PCR Kit (Qiagen) with Line-Gene K software (Bioer Technology Co. Ltd., Hangzhou, China). The $C_{\mathrm{T}}$ value for miR-1290 was normalized to U6 snRNA. The $2^{-\Delta \Delta C t}$ method was used to calculate relative expression level of miRNAs.

miRNA microarray analysis. Microarray analysis was performed using SurePrint G3 Human V16 miRNA 8x60K 
(Agilent, Santa Clara, CA, USA), according to the manufacturer's protocol. Briefly, $50 \mathrm{ng}$ total purified RNA was treated with calf intestine alkaline phosphatase and labeled with cyanine 3-cytidine bisphosphate using T4 RNA ligase. The labeled RNAs were hybridized to the probes on the microarray. The microarray data were analyzed using GeneSpring GX software version 11.5 (Agilent). The raw data were filtered using FLAG and t-test, and applied to the fold-change analysis. The fold-change analysis was conducted based on a factor of 2.0 -fold between two groups, DMSO-treated control cells and asiatic acid $(50 \mu \mathrm{M})$-treated cells.

Western blotting. Cells were collected and washed with cold phosphate-buffered saline (PBS). The cell pellets were lysed using modified radioimmunoprecipitation assay (RIPA) buffer (50 mM Tris, pH 8.0, $150 \mathrm{mM} \mathrm{NaCl}, 1 \% \mathrm{NP}-40,0.5 \%$ deoxycholate, $0.1 \%$ SDS) containing protease inhibitors (complete Tablets, Mini, EDTA-free, EASYpack; Roche Applied Science, Mannheim, Germany) at $4^{\circ} \mathrm{C}$ for $20 \mathrm{~min}$. The lysates were then centrifuged at $12,000 \mathrm{x}$ g for $30 \mathrm{~min}$. The supernatant was decanted and saved. The concentration of total protein was determined using Bradford assay (Bio-Rad). Fifty micrograms of total cellular protein were solubilized in SDS sample buffer and resolved by SDS-PAGE. Anti-BCL2 and anti$\beta$-actin primary antibodies were purchased from Santa Cruz Biotechnology (Santa Cruz, CA, USA) and Sigma-Aldrich, respectively.

\section{Results}

Characterization of asiatic acid-induced toxicity in A549 NSCLC cells. We first sought to determine whether asiatic acid, a triterpenoid derived from $C$. asiatica, affects the viability of A549 NSCLC cells. As shown in Fig. 1A, exposure of A549 cell cultures to concentrations of asiatic acid ranging from $0-100 \mu \mathrm{M}$ for $24 \mathrm{~h}$ induced cytotoxicity in a concentrationdependent manner, as determined by WST-1 assay. Maximal toxicity was obtained at a concentration of $100 \mu \mathrm{M}$, at which concentration cell viability was reduced to $18.63 \pm 0.13 \%$ of the control value $(\mathrm{n}=3)$. The $\mathrm{IC}_{50}$ (the concentration at which half maximal toxicity is observed) of asiatic acid was $50 \mu \mathrm{M}$; at this concentration, cell viability was reduced to $50.81 \pm 2.13 \%$ of control value (Fig. 1A).

We then examined the mechanism underlying asiatic acid-induced loss of viability in NSCLC A549 cells. Cells were treated with asiatic acid ranging from $0-100 \mu \mathrm{M}$ for $24 \mathrm{~h}$, stained with PI, and DNA content was analyzed by flow cytometry. As shown in Fig. 1B, asiatic acid caused a concentration-dependent increase in the proportion of cells in the sub-G1 phase, indicating that the effect of asiatic acid in NSCLC A549 cells is cell death-dependent. Although $25 \mu \mathrm{M}$ asiatic acid increased the proportion of cells in sub-G1 by only $3.67 \pm 0.16 \%$, concentrations of 50 and $100 \mu \mathrm{M}$ asiatic acid were associated with increases of $13.50 \pm 1.31 \%$ and $41.46 \pm 2.45 \%$, respectively. These results demonstrate that asiatic acid induces cell death-mediated loss of cell viability in NSCLC A549 cells.

Identification of miRNAs altered by asiatic acid treatment in NSCLC A549 cells. We used miRNA microarray analysis to

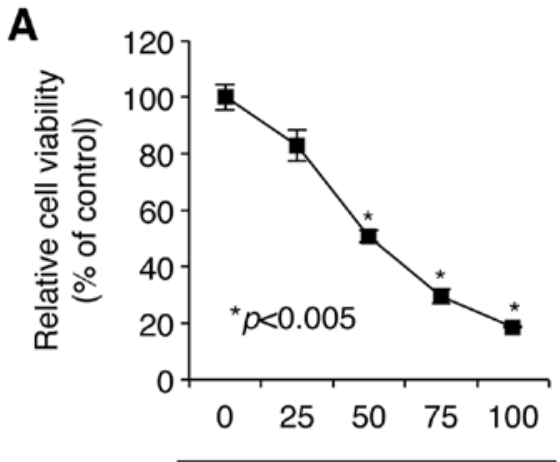

Asiatic acid $(\mu \mathrm{M})$

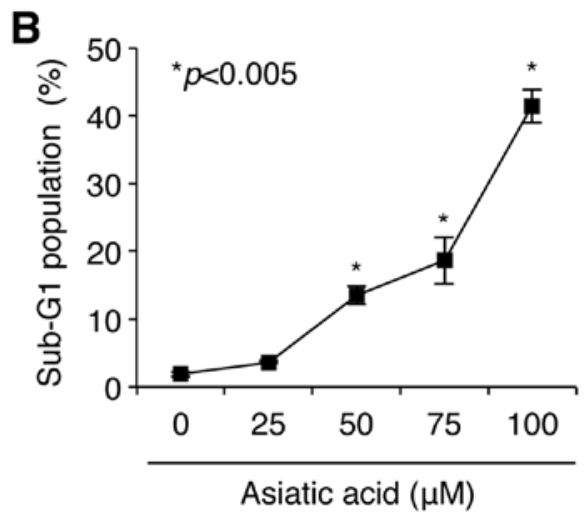

Figure 1. Asiatic acid induces cell death in A549 cells. (A) Asiatic acid reduced viability in A549 cells in a dose-dependent manner. Cells were seeded in 96-well culture dishes and treated with various doses of asiatic acid. After $24 \mathrm{~h}$ incubation, viabilities of the cells were measured by WST-1 assay. Each bar represents mean \pm SD from three independent experiments. ${ }^{*} \mathrm{P}<0.005$ compared with control. (B) Flow cytometric analysis showed that asiatic acid increased the sub-G1 apoptotic population of A549 cells. Cells were treated with different dose of asiatic acid for $24 \mathrm{~h}$. Cells were then fixed with ethanol and stained with PI solution. Cell cycle was evaluated using flow cytometry analysis. Values are mean $\pm \mathrm{SD}$ of three independent experiments. ${ }^{*} \mathrm{P}<0.005$ compared with the control.

investigate the effect of asiatic acid treatment of A549 cells on miRNA expression profiles. Exposure of A549 cells to $50 \mu \mathrm{M}$ asiatic acid increased or decreased $(\mathrm{P}<0.05)$ the expression levels of certain miRNAs by $>1.5$-fold (Fig. $2 \mathrm{~A}$ and Table I). Of these, 22 miRNAs were upregulated and 13 were downregulated (Fig. 2B). Notably, miR-1290, miR-1246, and miR-630 were significantly increased by 44.56-, 20.35-, and 9.11-fold, respectively, whereas expression of miR-503, miR-149-5p, and miR-126 were significantly downregulated by $2.53-, 2.47-$, and 2.33 -fold, respectively (Table I). These data suggest that the mechanism underlying asiatic acid-induced cell death is likely mediated by specific miRNAs. Notably, miR-1290 showed the greatest increase in expression with asiatic acid treatment. We, therefore, hypothesized that asiatic acid-induced cell death in NSCLS A549 cells may be related to the altered level of miR-1290 expression.

Upregulation of miR-1290 expression is associated with asiatic acid-induced cell death in NSCLC A549 cells. We next confirmed the asiatic acid-induced increase in miR-1290 level using quantitative RT-PCR with primers specific for 
Table I. miRNAs altered in response to asiatic acid treatment in A549 cells.

\begin{tabular}{|c|c|c|c|c|c|}
\hline $\operatorname{miRNA}^{\mathrm{a}}$ & $\mathrm{FC}^{\mathrm{b}}$ & Chromosome & miRNA & $\mathrm{FC}$ & Chromosome \\
\hline hsa-miR-1202 & 3.10 & 6 & hsa-miR-574-5p & 2.72 & 4 \\
\hline hsa-miR-1207-5p & 2.51 & 8 & hsa-miR-630 & 9.11 & 15 \\
\hline hsa-miR-1225-5p & 2.43 & 16 & hsa-miR-638 & 3.63 & 19 \\
\hline hsa-miR-1246 & 20.35 & 2 & hsa-miR-874 & 1.95 & 5 \\
\hline hsa-miR-1290 & 44.56 & 1 & hsa-miR-1234 & -1.57 & 8 \\
\hline hsa-miR-150-3p & 2.20 & 19 & hsa-miR-125a-5p & -1.53 & 19 \\
\hline hsa-miR-1915-3p & 2.23 & 10 & hsa-miR-126-3p & -2.33 & 9 \\
\hline hsa-miR-1973 & 1.96 & 4 & hsa-miR-149-5p & -2.47 & 2 \\
\hline hsa-miR-2861 & 3.36 & 9 & hsa-miR-181b-5p & -1.58 & 1 \\
\hline hsa-miR-3162-5p & 1.96 & 11 & hsa-miR-196a-5p & -1.52 & 12 \\
\hline hsa-miR-3196 & 1.91 & 20 & hsa-miR-210 & -1.52 & 11 \\
\hline hsa-miR-3656 & 3.23 & 11 & hsa-miR-22-5p & -1.53 & 17 \\
\hline hsa-miR-3665 & 1.79 & 13 & hsa-miR-425-5p & -1.59 & 3 \\
\hline hsa-miR-3679-5p & 4.08 & 2 & hsa-miR-455-3p & -1.52 & 9 \\
\hline hsa-miR-4281 & 2.36 & 5 & hsa-miR-484 & -1.56 & 16 \\
\hline hsa-miR-4298 & 3.79 & 11 & hsa-miR-503 & -2.53 & $\mathrm{X}$ \\
\hline hsa-miR-4299 & 1.88 & 11 & hsa-miR-98 & -1.77 & $X$ \\
\hline hsa-miR-494 & 2.44 & 14 & & & \\
\hline
\end{tabular}

${ }^{a}$ miRNAs showing $>1.5$-fold change in expression after flag sorting. ${ }^{b} \mathrm{FC}$, fold change.

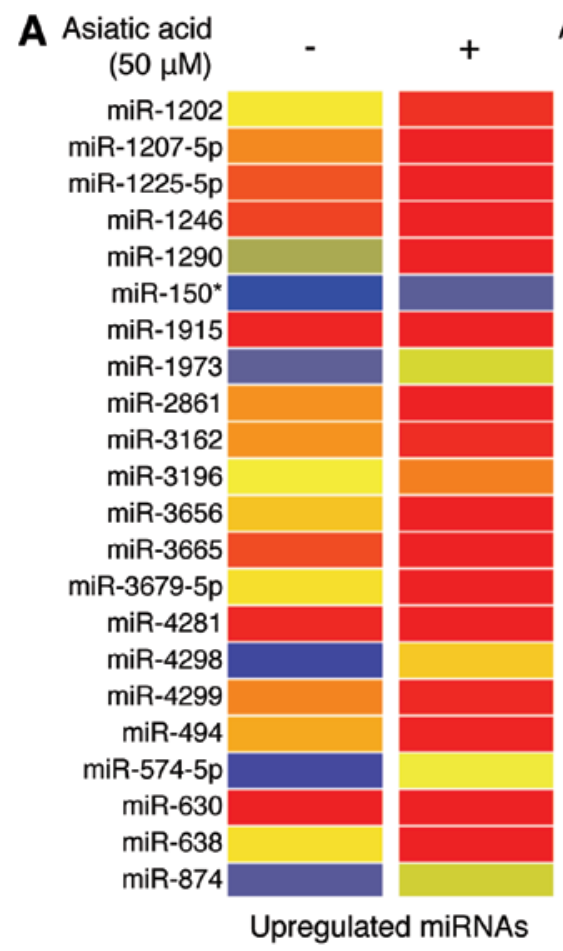

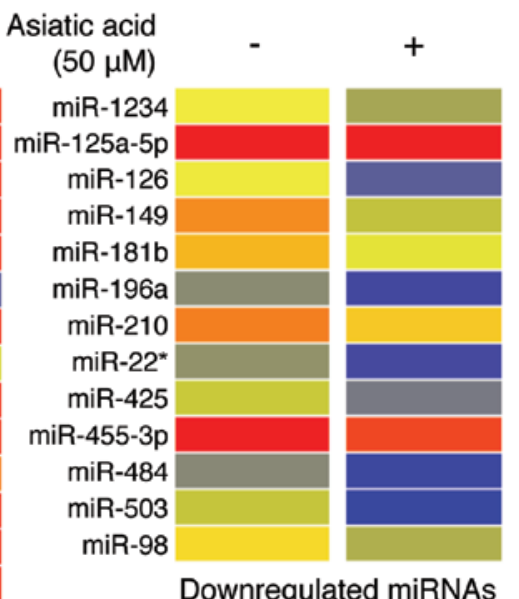

Downregulated miRNAs

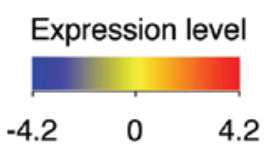

B

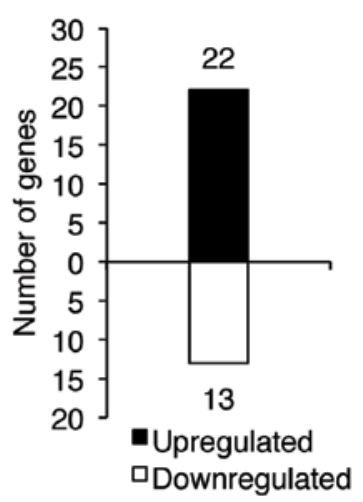

Figure 2. Asiatic acid alters miRNA expression in A549 cells. (A) Heat map showing results of miRNA microarray analysis of the effect of asiatic acid treatment on miRNA expression profiles in A549 cells. Cells were treated with asiatic acid (50 $\mu \mathrm{M})$ for $24 \mathrm{~h}$ and subjected to RNA extraction. miRNA microarray was performed to identify the changes in miRNA expression profiles. Upregulated (left panel) and downregulated (right panel) miRNAs by asiatic acid treatment in A549 cells are represented by a heat-map image. (B) The graph shows the number of miRNAs deregulated by asiatic acid treatment in A549 cells.

the mature miR-1290 sequence. As shown in Fig. 3A, exposure of A549 NSCLC cells to 25 and $50 \mu \mathrm{M}$ asiatic acid for $24 \mathrm{~h}$ induced large increases of 2.99-fold and 13.61-fold, respectively, in expression level of mature miR-1290. We then investigated the biological significance of miR-1290 upregulation in A549 cells in transient transfection experi- 

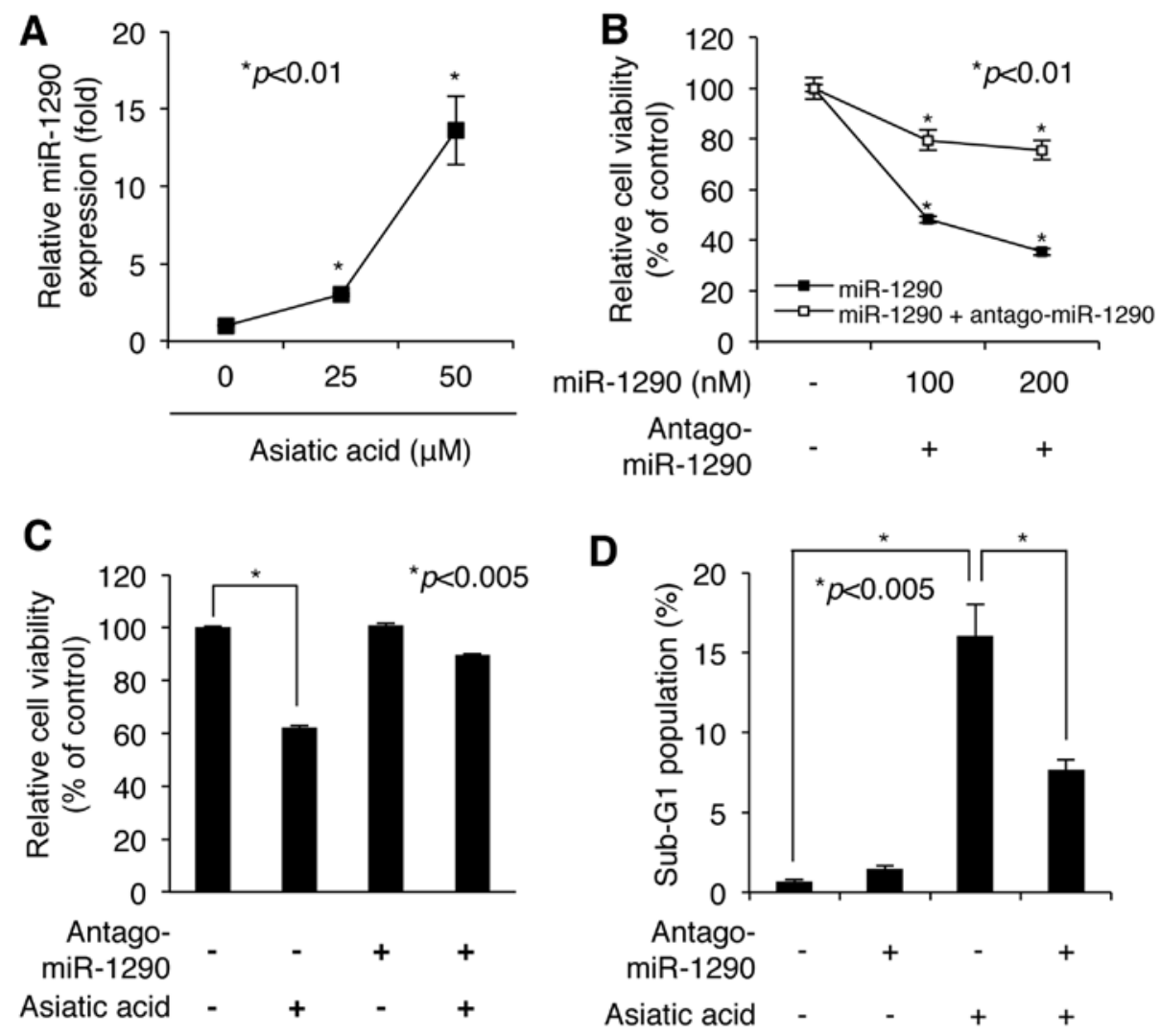

Figure 3. miR-1290 regulates asiatic acid-induced cell death in A549 cells. (A) Real-time PCR analysis of relative miR-1290 expression in A549 cells treated with the indicated amounts of asiatic acid. Cells were treated with different doses of asiatic acid $(0,25$ and $50 \mu \mathrm{M})$ for $24 \mathrm{~h}$ and subjected to RNA extraction. The level of miR-1290 was determined by real-time PCR. Values are presented as the mature-miR-1290 level normalized to the U6 snRNA level. The data are representative of three independent experiments (mean \pm SD). (B) Relative viability of A549 cells transfected with miR-1290 and equal amounts of its antagomiR. Cells were transfected with miR-1290 mimic and/or antagomiR-1290 for $24 \mathrm{~h}$. The viabilities of the cells were evaluated using WST-1 assay. Values are mean \pm SD of three independent experiments. "P<0.01 compared with the control. (C) Relative viability of A549 cells transfected with antagomiR-1290 and treated with asiatic acid. Cells were transfected with antagomiR-1290 and then treated with asiatic acid for $24 \mathrm{~h}$. Relative cell viabilities were evaluated using WST-1 assay. (D) Flow cytometric analysis of relative size of the sub-G1 apoptotic population of A549 cells transfected with antagomiR-1290 and treated with asiatic acid. Cells were transfected with antagomiR-1290 for $24 \mathrm{~h}$ and then treated with asiatic acid $(50 \mu \mathrm{M})$. After $24 \mathrm{~h}$ of incubation, the cells were subjected to flow cytometry. The percentage of sub-G1 cells is indicated in the graph. Values are mean \pm SD of three independent experiments. "P< $<0.005$ compared with the control.

ments. As shown in Fig. 3B, A549 cells transfected with 100 and $200 \mathrm{nM}$ miR-1290 mimic underwent loss of viability in a dose-dependent manner, as assessed by WST-1 assay. Maximal loss of cell viability was obtained at a concentration of $200 \mathrm{nM}$ miR-1290 mimic, which decreased cell viability to $35.35 \pm 1.2 \%$ of the control value ( $n=3)$ (Fig. 3B). However, inhibition of miR-1290 activity by antagomiR-1290 blocked most of the miR-1290-induced decrease in cell viability (Fig. 3B). Notably, cotransfection of $200 \mathrm{nM}$ miR-1290 mimic and an equal amount of its antagomiR showed $75.49 \pm 3.76 \%(n=3)$ of control cell viability, whereas transfection with miR-1290 mimic alone showed $35.35 \pm 1.2 \%$ of control viability (Fig. 3B). These results indicate that miR-1290 upregulation could be involved in cell growth defect.

Next, we further evaluated whether asiatic acid-induced loss of cell viability is dependent on miR-1290 upregulation. Prior to exposure of NSCLC A549 cells to asiatic acid (50 $\mu \mathrm{M})$, cells were transfected with antagomiR-1290 (200 nM) for $24 \mathrm{~h}$. As shown in Fig. 3C, cells transfected with the antagomiR showed resistance to asiatic acid-induced cytotoxicity. Flow cytometric analysis after staining with PI also showed that asiatic acid-induced cell death was significantly attenuated in the presence of antagomiR-1290 (Fig. 3D). Although exposure of control cells to asiatic acid $(50 \mu \mathrm{M})$ showed a $16.05 \pm 1.99 \%$ increase in the proportion of the cell population in sub-G1 phase, exposure of antagomiR-1290-transfected cells to asiatic acid showed only a $7.69 \pm 0.59$ increase. These results demonstrate that asiatic acid-induced cell death is mediated by accumulation of miR-1290 in NSCLC A549 cells.

miR-1290 upregulated by asiatic acid targets BCL2 mRNA in NSCLC A549 cells. Previous studies showed that asiatic acid induced cell growth inhibition by decreasing the level of BCL2 protein in MCF7 human breast cells and HT-29 human colon adenocarcinoma cells $(7,8)$. We also evaluated the effect of asiatic acid on the levels of BCL2 in A549 cells. Asiatic acid strongly decreased intracellular levels of BCL2 protein, as revealed by western blotting (Fig. 4A). We next investigated whether there was a correlation between the reduced level of BCL2 and increased miR-1290 expression in asiatic acid-treated A549 cells. We first used bioinformatics analysis to analyze the possibility that miR-1290 targets BCL2 mRNA. As shown in Fig. 4B, miR-1290 contains a sequence complementary sequence to the 3'-UTR of human BCL2 
A

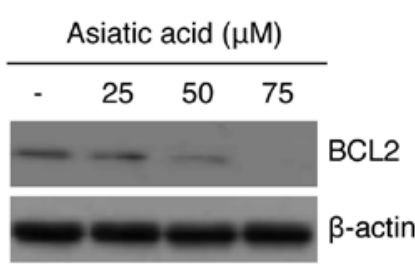

B

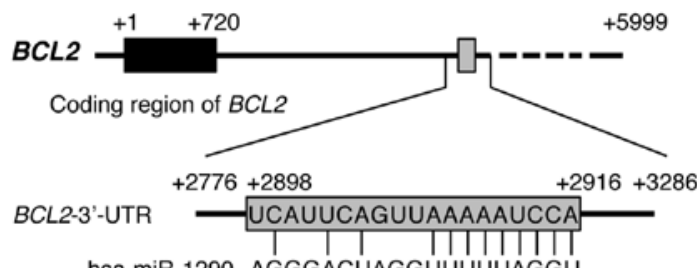

hsa-miR-1290 AGGGACUAGGUUUUUAGGU

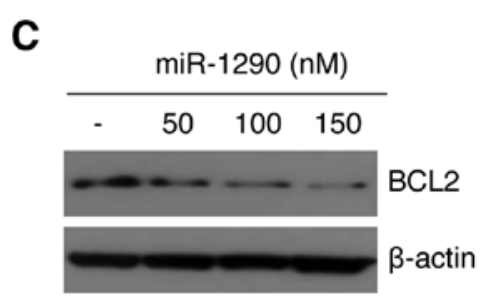

E

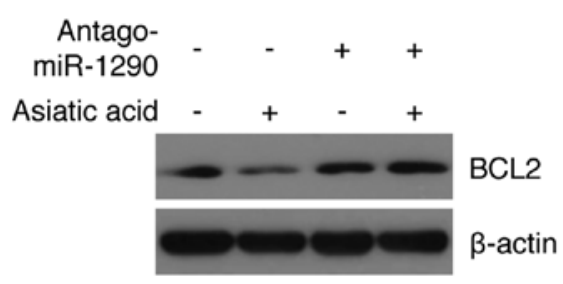

D miR-1290 (nM) - - - $\quad \begin{array}{llll}50 & 100 & 100\end{array}$

Antago-miR-1290 - $50100 \quad-\quad$ - 100

(nM)

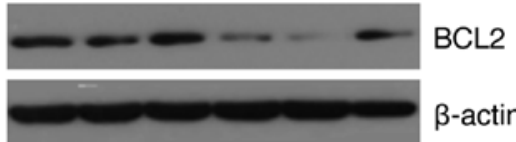

$\mathbf{F}$

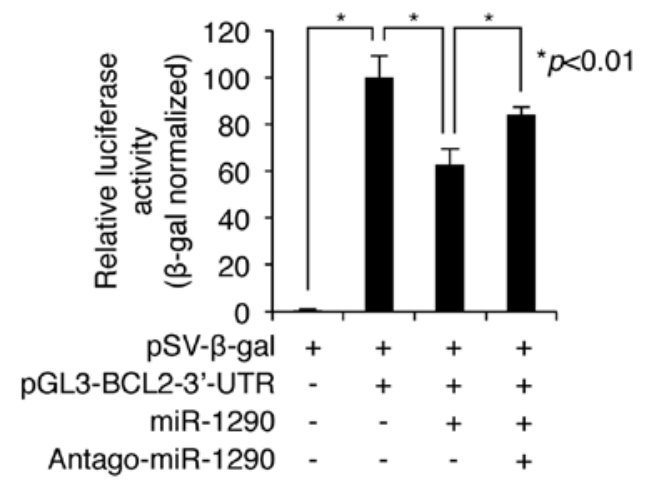

Figure 4. miR-1290 negatively regulates BCL2 expression in asiatic acid-treated A549 cells. (A) Western blot analysis of BCL2 expression in A549 cells treated with the indicated amounts of asiatic acid. $\beta$-actin served as internal control. A549 cells were treated with different doses of asiatic acid $(0,25$ and $50 \mu \mathrm{M})$ for $24 \mathrm{~h}$. Cells were collected and subjected to western blotting using anti-BCL2 and anti- $\beta$-actin antibodies. Anti- $\beta$-actin antibody was used as a loading control in experiments. (B) In silico analysis of $B C L 2 \mathrm{mRNA}$ and the predicted target sequence of miR-1290. ATG start codon is indicated by +1 . The $3{ }^{\prime}$ end of $B C L 2$ coding region is indicated by +720 . The sequences of the 3-'UTR containing miR-1290 recognition sequences are located from +2776 to +3286 in human BCL2 mRNA, respectively. Gray box (+2886 to +2916) indicates target-specific binding site of miR-1290 in 3'-UTR of BCL2 mRNA. (C) Western blot analysis of BCL2 expression in A549 cells transfected with the indicated amounts of miR-1290. $\beta$-actin served as internal control. Different concentrations of miR-1290 mimic $(0,50,100$ and $150 \mathrm{nM})$ were transfected into A549 cells. After $24 \mathrm{~h}$ of incubation, the levels of BCL2 proteins were analyzed by western blotting with anti-BCL2-specific antibody. (D) Western blot analysis of BCL2 expression in A549 cells transfected with the indicated amounts of miR-1290 and its antagomiR. $\beta$-actin served as internal control. AntagomiR-1290 inhibited miR-1290-mediated BCL2 downregulation. A549 cells were transfected with miR-1290 and/or antagomiR-1290 as indicated above. After $24 \mathrm{~h}$ of incubation, the cells were collected and subjected to western blotting using anti-BCL2 and anti- $\beta$-actin antibody. (E) Western blot analysis of BCL2 expression in A549 cells transfected with miR-1290 or control miRNA and incubated in the presence or absence of asiatic acid. $\beta$-actin served as internal control. AntagomiR-1290 impeded asiatic acid-induced BCL2 decrease. A549 cells were transfected with antagomiR-1290 $(150 \mathrm{nM})$ for $24 \mathrm{~h}$. Then, asiatic acid $(50 \mu \mathrm{M})$ was treated into the cells. After $24 \mathrm{~h}$ of incubation, the levels of BCL2 proteins were evaluated by western blotting using anti-BCL2 antibody. (F) BCL2 is a putative target of miR-1290. Relative luciferase activity in cells transfected with reporter construct containing the miR-1290 recognition sequences of the BCL2 gene (pGL-BCL2-3'-UTR), miR-1290 mimic, antagomiR-1290, and pSV- $\beta$-galactosidase normalization vector, as indicated. After $24 \mathrm{~h}$ of incubation, cells were collected and subjected to luciferase assay. Values are mean \pm SD of three independent experiments. ${ }^{*} \mathrm{P}<0.01$ compared with the control.

mRNA. The predicted target site of miR-1290 is encoded by nucleotides 2898-2916 in the 3'-UTR (Fig. 4B). We therefore examined whether miR-1290 reduces BCL2 protein levels in NSCLC A549 cells. As shown in Fig. 4C, exposure of cells to miR-1290 mimic (50, 100, and $150 \mathrm{nM})$ significantly decreased BCL2 level in a dose-dependent manner. We also found that the decrease in BCL2 was largely blocked by antagomiR-1290 (Fig. 4D). In addition, we sought to determine whether the downregulation of BCL2 by asiatic acid treatment was affected by inhibition of miR-1290 activity. Notably, the asiatic acidinduced decrease in BCL2 decrease was largely rescued in antagomiR-1290-transfected cells (Fig. 4E). To assess whether BCL2 is a direct target of miR-1290, we constructed recombinant luciferase plasmids in which the 3-UTR of BCL2 is cloned downstream of the firefly luciferase reporter gene in pGL3 vectors. The reporter plasmid was cotransfected with either the miR-1290 mimic or its antagomiR in NSCLC A549 cells, and luciferase activity was measured $24 \mathrm{~h}$ after transfection. As shown in Fig. 4F, overexpression of miR-1290 significantly reduced the luciferase activity of pGL3-BCL23-UTR to $62.71 \pm 6.72 \%(n=3)$. Conversely, this downregulation was rescued by cotransfection with antagomiR-1290 (Fig. 4F). These results indicate that miR-1290 directly targets the 3'-UTR of BCL2 mRNA, and negatively regulates BCL2 expression post-transcriptionally.

\section{Discussion}

The present study examined the role of miR-1290 in the negative regulation of BCL 2 expression in NSCLC A549 cells. The expression level of miR-1290 was significantly increased by asiatic acid, as revealed by miRNA microarray and quantita- 
tive RT-PCR analyses. Although we did not examine whether asiatic acid increases transcription of miR 1290 or enhances its stability, this increase may be mediated at the transcriptional level. First, the microarray data indicated alterations in the expression of specific miRNAs. Second, the level of up- or downregulation of these miRNAs varied significantly. Third, although several miRNAs, including miR-382 and miR-154, show differential stability, most miRNAs appear to be stable in human cells (23). Additionally, our microarray data showed that only miR-1290 was significantly elevated (by 44-fold), whereas other miRNAs showed a trend toward increased or decreased levels, without reaching a similar level of fold change.

We found that the interplay between asiatic acid and cell death depends on the expression level of miR-1290, which tightly regulates asiatic acid-induced cell death. Transient transfection of A549 cells with miR-1290 mimics downregulated BCL2 expression and increased cell growth inhibition, as well as cell death. MiR-1290-dependent cell death was also investigated using ectopic expression of antagomiR-1290 oligonucleotides. WST-1 assay and FACS analysis revealed that asiatic acid-induced cell death was largely blocked by inhibition of miR-1290 activity. Furthermore, antagomiR-1290 inhibited asiatic acid-induced BCL2 downregulation. Collectively, our data indicate that asiatic acid-induced cell death is miR-1290 upregulation-dependent.

Currently, the effect of miR-1290 on growth inhibition and cell death is a matter of controversy. Some authors have suggested that miR-1290 is significantly upregulated in clinical colon cancer tissues and in the serum of patients with pancreatic cancer, compared to healthy controls $(24,25)$. However, others argue that miR-1290 is strongly downregulated in clear renal cell carcinoma tissue and hypermethylated in cervical cancer cell lines $(26,27)$. Similarly, our previous reports showed that miR-1290 is significantly upregulated by 2 -fold in response to ginsenoside $\mathrm{Rh} 2$, which induces cell death in NSCLC A549 cells (28). Also our unpublished data show that asiatic acid induced cell death in MCF7 human breast cancer and AGS human gastric cancer cells, and upregulated expression of miR-1290 by 2.58- and 7.01-fold, respectively. Recently, introduction of miR-1290 into estrogen receptor $\alpha$-positive breast cancer cells and neuroblastoma cells decreased expression of NAT and FOXA1 and led to a slowing down of the cell cycle $(29,30)$. Collectively, these results indicate that miR-1290 functions as a putative tumor suppressor in breast, lung and gastric cancer by inducing cell cycle arrest and apoptosis; however, it has a oncogenic function in colon and pancreatic cancer. The data indicate that miR-1290 has a dual role as tumor suppressor and oncogene in a cell-typespecific manner, although further investigation is needed to elucidate the precise mechanisms underlying these functions.

BCL2, a well-known pro-survival protein localized in mitochondria, has been reported to have antiapoptotic properties $(31,32)$. In particular, the antiapoptotic function of BCL2 is based on its ability to inhibit an apoptotic mechanism induced by several anticancer agents, such as paclitaxel and cisplatin $(33,34)$, and BCL2 downregulation has been observed in almost all apoptotic processes (35). Recent studies have shown that asiatic acid induces apoptosis and cell cycle arrest through BCL2 downregulation in human breast cancer cells and human colon adenocarcinoma cells $(7,8)$. Also, asiatic acid-induced BCL2 downregulation was attenuated by pretreatment with ERK1/2 inhibitor U0216 in breast cancer cells (8). However, the mechanism underlying BCL2 downregulation induced by asiatic acid treatment of cells remains unknown. Here, we showed that asiatic acid-induced BCL2 downregulation was due to miR-1290-mediated posttranscriptional inhibition of BCL2 mRNA. In silico analysis showed that the 3'-UTR of BCL2 mRNA contains a complementary binding site for miR-1290, and luciferase assay confirmed the implication of BCL2 mRNA as a direct target of miR-1290. Endo et al showed other putative target mRNAs of miR-1290 (29). They demonstrated that NAT and FOXA1 proteins are downregulated by miR-1290 overexpression; however, it remains unknown whether these genes are direct targets of miR-1290 (29). Therefore, BCL2 is the first confirmed target mRNA of miR-1290 in cells.

In summary, we determined that asiatic acid induced growth inhibition and death in A549 NSCLC cells. We also showed that asiatic acid induces considerable changes in the miRNA expression profile of the cells. Notably, miR-1290 is the most extensively upregulated miRNA, and it tightly regulates asiatic acid-induced cell death. Further experiments demonstrated that miR-1290 directly regulates BCL2 synthesis. Our results may provide a useful approach to understanding cellular responses to asiatic acid, although the clinical significance of miR-1290 and BCL2 warrant further investigation.

\section{Acknowledgements}

We would like to thank all members of our laboratory for their support and advice during this study. This study resulted from the Konkuk University research support program.

\section{References}

1. Howes MJ and Houghton PJ: Plants used in Chinese and Indian traditional medicine for improvement of memory and cognitive function. Pharmacol Biochem Behav 75: 513-527, 2003.

2. Hausen BM: Centella asiatica (Indian pennywort), an effective therapeutic but a weak sensitizer. Contact Dermatitis 29: 175-179, 1993.

3. Rush WR, Murray GR and Graham DJ: The comparative steadystate bioavailability of the active ingredients of Madecassol. Eur J Drug Metab Pharmacokinet 18: 323-326, 1993.

4. Xiong Y, Ding H, Xu M and Gao J: Protective effects of asiatic acid on rotenone- or $\mathrm{H}_{2} \mathrm{O}_{2}$-induced injury in SH-SY5Y cells. Neurochem Res 34: 746-754, 2009.

5. Ma K, Zhang Y, Zhu D and Lou Y: Protective effects of asiatic acid against D-galactosamine/lipopolysaccharide-induced hepatotoxicity in hepatocytes and kupffer cells co-cultured system via redox-regulated leukotriene $\mathrm{C} 4$ synthase expression pathway. Eur J Pharmacol 603: 98-107, 2009.

6. Lee YS, Jin DQ, Kwon EJ, et al: Asiatic acid, a triterpene, induces apoptosis through intracellular $\mathrm{Ca}^{2+}$ release and enhanced expression of p53 in HepG2 human hepatoma cells. Cancer Lett 186: 83-91, 2002.

7. Bunpo $\mathrm{P}$, Kataoka K, Arimochi $\mathrm{H}$, et al: Inhibitory effects of asiatic acid and CPT-11 on growth of HT-29 cells. J Med Invest 52: 65-73, 2005.

8. Hsu YL, Kuo PL, Lin LT and Lin CC: Asiatic acid, a triterpene, induces apoptosis and cell cycle arrest through activation of extracellular signal-regulated kinase and p38 mitogen-activated protein kinase pathways in human breast cancer cells. J Pharmacol Exp Ther 313: 333-344, 2005.

9. Sastry PS and Rao KS: Apoptosis and the nervous system. J Neurochem 74: 1-20, 2000.

10. Nakano R: Apoptosis: gene-directed cell death. An overview. Horm Res 48: 2-4, 1997. 
11. Moldoveanu T, Follis AV, Kriwacki RW and Green DR: Many players in BCL-2 family affairs. Trends Biochem Sci 39: 101-111, 2014.

12. Cheng AM, Byrom MW, Shelton J and Ford LP: Antisense inhibition of human miRNAs and indications for an involvement of miRNA in cell growth and apoptosis. Nucleic Acids Res 33: 1290-1297, 2005.

13. He L, He X, Lim LP, et al: A microRNA component of the p53 tumour suppressor network. Nature 447: 1130-1134, 2007.

14. Bommer GT, Gerin I, Feng Y, et al: p53-mediated activation of miRNA34 candidate tumor-suppressor genes. Curr Biol 17: 1298-1307, 2007.

15. Christoffersen NR, Shalgi R, Frankel LB, et al: p53-independent upregulation of miR-34a during oncogene-induced senescence represses MYC. Cell Death Differ 17: 236-245, 2010.

16. Liu C, Yu J, Yu S, et al: MicroRNA-21 acts as an oncomir through multiple targets in human hepatocellular carcinoma. J Hepatol 53: 98-107, 2010.

17. Ma X, Kumar M, Choudhury SN, et al: Loss of the miR-21 allele elevates the expression of its target genes and reduces tumorigenesis. Proc Natl Acad Sci USA 108: 10144-10149, 2011.

18. Papagiannakopoulos T, Shapiro A and Kosik KS: MicroRNA-21 targets a network of key tumor-suppressive pathways in glioblastoma cells. Cancer Res 68: 8164-8172, 2008

19. Yao Q, Cao S, Li C, Mengesha A, Kong B and Wei M Micro-RNA-21 regulates TGF- $\beta$-induced myofibroblast differentiation by targeting PDCD4 in tumor-stroma interaction. Int J Cancer 128: 1783-1792, 2011

20. Medina PP, Nolde $M$ and Slack FJ: OncomiR addiction in an in vivo model of microRNA-21-induced pre-B-cell lymphoma. Nature 467: 86-90, 2010.

21. An IS, An S, Kang SM, et al: Titrated extract of Centella asiatica provides a UVB protective effect by altering microRNA expression profiles in human dermal fibroblasts. Int J Mol Med 30: 1194-1202, 2012.

22. An IS, An S, Choe TB, et al: Centella asiatica protects against UVB-induced HaCaT keratinocyte damage through microRNA expression changes. Int J Mol Med 30: 1349-1356, 2012.

23. Bail S, Swerdel M, Liu H, et al: Differential regulation of microRNA stability. RNA 16: 1032-1039, 2010.

24. Wu J, Ji X, Zhu L, et al: Up-regulation of microRNA-1290 impairs cytokinesis and affects the reprogramming of colon cancer cells. Cancer Lett 329: 155-163, 2013.
25. Li A, Yu J, Kim H, et al: MicroRNA array analysis finds elevated serum miR-1290 accurately distinguishes patients with low-stage pancreatic cancer from healthy and disease controls. Clin Cancer Res 19: 3600-3610, 2013.

26. White NM, Bao TT, Grigull J, et al: miRNA profiling for clear cell renal cell carcinoma: biomarker discovery and identification of potential controls and consequences of miRNA dysregulation. J Urol 186: 1077-1083, 2011.

27. Yao T, Rao Q, Liu L, et al: Exploration of tumor-suppressive microRNAs silenced by DNA hypermethylation in cervical cancer. Virol J 10: 175, 2013

28. An IS, An S, Kwon KJ, Kim YJ and Bae S: Ginsenoside Rh2 mediates changes in the microRNA expression profile of human non-small cell lung cancer A549 cells. Oncol Rep 29: 523-528, 2013.

29. Endo Y, Toyama T, Takahashi S, et al: miR-1290 and its potential targets are associated with characteristics of estrogen receptor $\alpha$-positive breast cancer. Endocr Relat Cancer 20: 91-102, 2013

30. Yelamanchili SV, Morsey B, Harrison EB, et al: The evolutionary young miR-1290 favors mitotic exit and differentiation of human neural progenitors through altering the cell cycle proteins. Cell Death Dis 5: e982, 2014

31. Kluck RM, Bossy-Wetzel E, Green DR and Newmeyer DD: The release of cytochrome $c$ from mitochondria: a primary site for Bcl-2 regulation of apoptosis. Science 275: 1132-1136, 1997.

32. Yang J, Liu X, Bhalla K, et al: Prevention of apoptosis by Bcl-2: release of cytochrome $\mathrm{c}$ from mitochondria blocked. Science 275: 1129-1132, 1997.

33. Gazitt Y, Rothenberg ML, Hilsenbeck SG, Fey V, Thomas C and Montegomrey W: Bcl-2 overexpression is associated with resistance to paclitaxel, but not gemcitabine, in multiple myeloma cells. Int J Oncol 13: 839-848, 1998.

34. Cho HJ, Kim JK, Kim KD, et al: Upregulation of Bcl-2 is associated with cisplatin-resistance via inhibition of Bax translocation in human bladder cancer cells. Cancer Lett 237: 56-66, 2006.

35. Cory S and Adams JM: The Bcl2 family: regulators of the cellular life-or-death switch. Nat Rev Cancer 2: 647-656, 2002.

36. Choi YM, An S, Lee EM, et al: CYP1A1 is a target of miR892a-mediated post-transcriptional repression. Int J Oncol 41: 331-336, 2012. 